\title{
Emittance compensation schemes for a superconducting rf injector
}

\author{
H. Vennekate, ${ }^{1,2,}$ A. Arnold, ${ }^{2}$ P. Lu, ${ }^{2}$ P. Murcek, ${ }^{2}$ J. Teichert, ${ }^{2}$ and R. Xiang ${ }^{2}$ \\ ${ }^{1}$ RI Research Instruments GmbH, Friedrich-Ebert-Straße 75, 51429 Bergisch Gladbach, Germany \\ ${ }^{2}$ Helmholtz-Zentrum Dresden-Rossendorf, Bautzner Landstraße 400, 01328 Dresden, Germany
}

(Received 22 December 2017; published 18 September 2018)

\begin{abstract}
Contemporary particle injector technologies provide different advantages depending on the chosen design. In the case of copper rf injectors these is primarily the high accelerating field, enabling the generation of high charge bunches with very low emittance. However, the cost of that is a comparably low repetition rate. DC guns, on the other hand, can provide higher repetition rates and consequently increased beam currents at lower beam quality, i.e., increased emittance. The concept of a superconducting rf injector offers the opportunity to combine the advantages of both these concepts. However, it demands special concepts for emittance compensation, as the common approach with overlapping magnetic fields during the rf acceleration interferes with the limitations of superconductivity. The ELBE SRF Gun project is one of the most advanced in this field. Gun II, the second SRF injector at the Electron Linear accelerator with high Brilliance and low Emittance (ELBE), introduces new features for emittance compensation which were studied in detail over the last years. One of these methods is the integration of a superconducting solenoid into the cryostat. Another method uses rf focusing by retracting the photocathode's tip from the last cell of the resonator. This paper discusses both of these schemes by briefly outlining their setups, discussing results of numerical simulations of their impact, and presenting results of initial experimental beam measurements with Gun II.
\end{abstract}

DOI: 10.1103/PhysRevAccelBeams.21.093403

\section{INTRODUCTION}

Many future accelerator concepts, such as energy recovery linacs (ERLs) and high power free electron lasers (FELs), demand injectors capable of delivering high bunch charge at increased repetition rates. Consequently, the development of superconducting radio frequency (SRF) electron injectors is of great interest [1-8]. Among these projects, the ELBE SRF Gun is one of the earliest and most advanced, running since the late 1990s. The ELBE accelerator facility at Helmholtz-Zentrum Dresden-Rossendorf (HZDR) in Dresden, Germany, offers a unique test environment as a $\mathrm{CW}$ machine, driving two FELs and several other secondary sources, which is ideal for the development and implementation of such a new particle source [9]. Besides feeding the current accelerator, the SRF Gun is expected to be the injector for the proposed Dresden Advanced Light Infrastructure and is furthermore embedded in the Helmholtz-Alliance's Gun cluster, cooperating for example with the BERLinPro project at HZB in Berlin.

\footnotetext{
*hannes.vennekate@ research-instruments.de

Published by the American Physical Society under the terms of the Creative Commons Attribution 3.0 License. Further distribution of this work must maintain attribution to the author(s) and the published article's title, journal citation, and DOI.
}

The currently installed injector is the second, updated version of the 3.5 cell niobium cavity photo electron source, operating with normal conducting photocathodes and a UV drive laser at $262 \mathrm{~nm}$ wavelength. Compared to Gun I, the field distribution inside the first half cell of the resonator has changed from $60 \%$ to $80 \%$ of the peak field in the $\pi$-mode. Besides this improved resonator, the cryostat itself was extended in order to house an additional superconducting (SC) solenoid for emittance compensation of the accelerated electron bunches, see Fig. 1, [10,11].

Since spring 2015 a transfer system was added to the injector, making it possible to exchange the cathodes

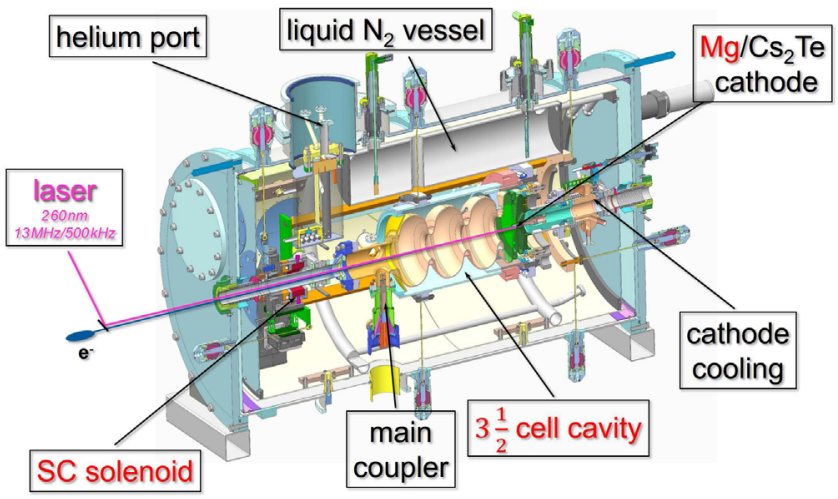

FIG. 1. Overview of the design of the ELBE SRF Gun II. 




FIG. 2. Example of the transverse electrical field lines affecting the cathode tip. In the indicated area the effect of $\mathrm{rf}$ focusing becomes visible, as the field lines indicate the additional focus toward the central gun axis for a retracted cathode.

without warming up any part of the cryostat. Besides the initial copper cathode, used during the commissioning phase, the SRF Gun II has been operated with bulk magnesium and $\mathrm{Cs}_{2} \mathrm{Te}$ coated photocathodes [12].

\section{RF FOCUSING}

The idea of rf focusing is based on the geometry of the gun cavity. Since the cathode is thermally isolated from the niobium resonator, it must be inserted into the first half cell through a narrow tube. When synchronized correctly to the laser phase, the standing longitudinal electric field accelerates the emitted electrons at the peak of the cycle and accelerates them into the injector beamline. Even when retracted by just a few microns, the electric field lines at the photocathode's tip have a focusing effect. Electrons emitted (further) away from the cathode's center, receive an additional kick towards the gun axis, as illustrated in simulations generated with the SUPERFISH code, shown in Fig. 2 $[13,14]$.

This early electron lens does not come without any cost. The retracted photocathode does not see the full field of the half cell, since parts of the electric field gradient is utilized for the focusing.

Since the functional tip of any photocathode in the ELBE SRF Gun is set at the end of a nominally $10 \mathrm{~cm}$ long copper body, the simplest approach to affect its location inside the cavity is to increase/decrease the length of the body's neck before inserting it into the cavity. This option can be utilized to generate larger offsets for retraction. A more precise mechanism for fine control of the cathode position has been realized inside the gun itself. The device referred to as "cathode tuner," depicted in Fig. 3, consists of three threaded studs inside the cryostat, which are connected to

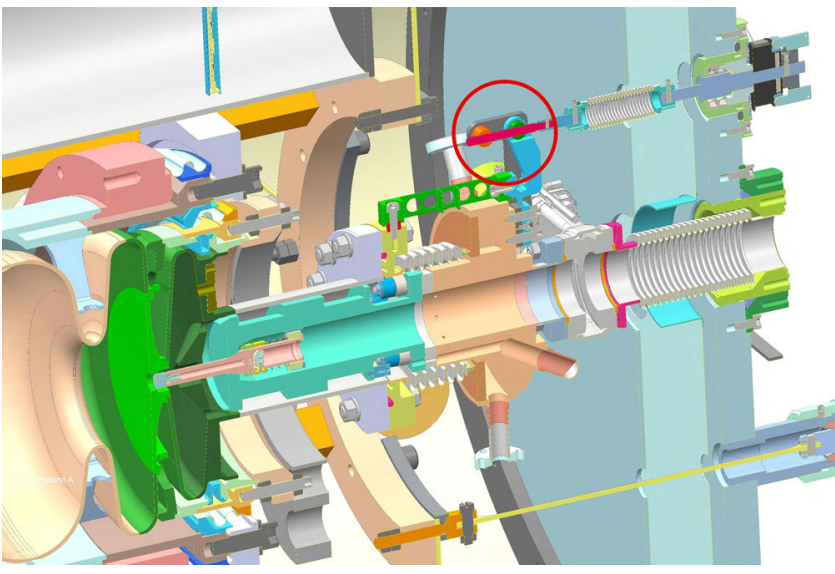

FIG. 3. CAD image for the cathode tuner, half cell (green) of the resonator and the cathode cooler inside the gun cryostat. The indicated mechanism connects the cathode cooler/holder to a gear outside the cryostat, making it possible to move the cathode tip in and out of the resonator during operation. The green bridge left and below of the highlighted area is, besides the mechanical connection, also responsible for the thermal decoupling of the cathode cooler and tuner.

three gears outside the cryostat's lid. The latter ones are linked to each other via a cam belt. Using a stepper motor and specially adapted transmission, the belt can be set in motion, simultaneously moving all three gears, and hence the three studs on the inside. As a result the entire cathode cooler, holding the installed photocathode, translates along the beam axis. The total range of this mechanism corresponds to a movement of $1.2 \mathrm{~mm}$ of the cathode with a stepper resolution of $1 \mu \mathrm{m}$.

As mentioned earlier, the niobium resonator is made superconducting by cooling with liquid helium. The cathode, on the other hand, is only cooled with liquid nitrogen, staying at about $77 \mathrm{~K}$, while the cathode tuner remains at room temperature as it is thermally linked to the outside of the cryostat. These different temperatures at different parts of the gun, together with all other uncertainties during installation, make it rather difficult to predict the exact location of the cathode's front surface within the injector when put into operation. One indirect way to determine the position of the cathode with respect to the half cell, is to measure its influence on the resonance frequency of the cavity for different positions of the cathode tuner and compare the results to simulation. This method yields a useful (rough) estimate of the displacement, as shown by A. Arnold in [15]. Recently, a setup for a direct measurement was developed [16]. It combines a linear and a rotary stage to align the line of sight onto the cathode, carrying a laser driven distance measuring device, and can be placed in the laser beamline of the gun. During beam generation the drive laser is reflected onto the photocathode in the half cell via a vacuum mirror inside the beam pipe. The very same mirror, which is placed 


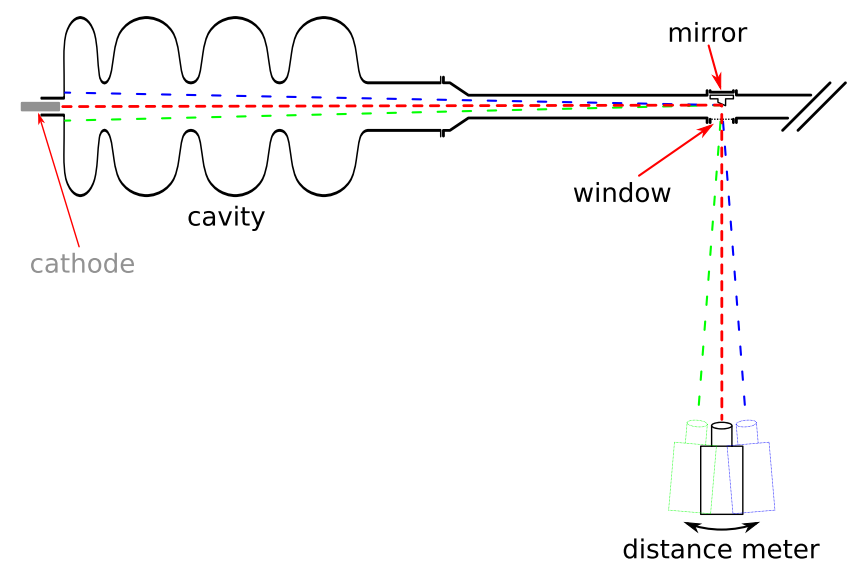

FIG. 4. Scheme of the experimental setup of the cathode localization: The laser driven distance meter uses the same mirror, which is used to shine UV light on the photocathode during operation, to scan the cathode tip with respect to the half cell's back plate.

slightly off the pipe's center to allow for the electron bunches to pass by, enables the laser distance measurement system to scan both, the cathode and the back plate of the half cell next to it, as shown in Fig. 4.

Subtracting the measured distance of the half cell's back plate from the result for the cathode tip, returns the actual retraction. The result of such a measurement of the cathode at the default central position of the cathode tuner is shown in Fig. 5.

The combined results of two measurements locate the copper cathode's tip at $z_{c}=-1.56 \pm 0.02 \mathrm{~mm}$ with respect to the half cell's rear plate being at $z_{0}=0$ on the beam axis.

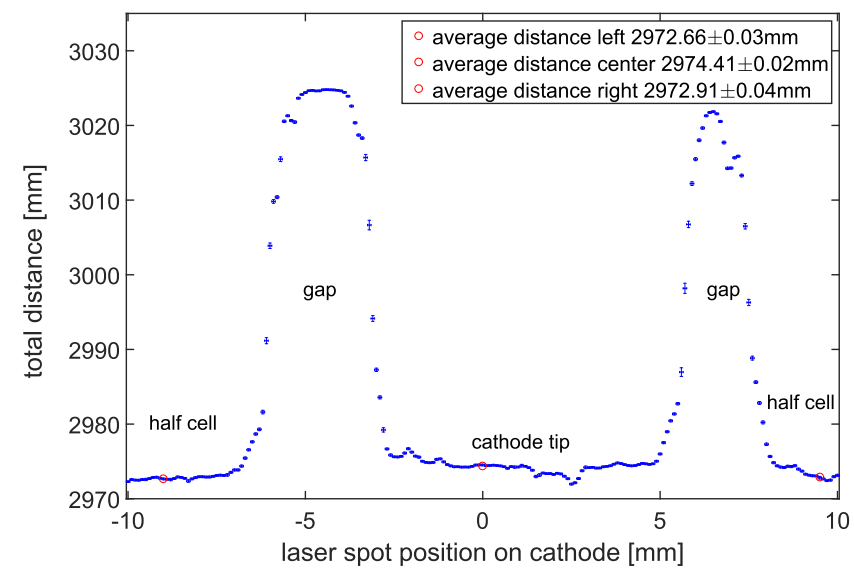

FIG. 5. Result of a cathode localization measurement. The distance values on the left and right of the center represent the distance of the measuring device to the half cell around the cathode, while the middle part represents the distance to the cathode's front tip. Since the size of the scanning laser spot is on the order of 2-3 mm the gaps in between cathode and half cell seem larger than they are in reality $(<1 \mathrm{~mm})$.

\section{SUPERCONDUCTING SOLENOID}

SRF Gun I at HZDR included a large, normal conducting solenoid directly at the cryomodule output in $\mathrm{z}$ direction, to alleviate the emittance growth down the beamline. This solenoid was located at roughly $1.1 \mathrm{~m}$ from the cathode. For the increased bunch charge aimed for with SRF Gun II, the magnetic lens was moved inside the cryostat and hence closer to the cathode. The new solenoid utilizes superconducting windings, is hence much smaller and is mounted directly in front of the cavity, as shown schematically in Fig. 6, placing it at about $0.7 \mathrm{~m}$ from the cathode.

The superconducting solenoid itself consists of a magnetic coil, made out of NbTi wires inside a copper matrix with about 2000 windings, and an iron yoke, both manufactured by NiOWAVE [17]. An exploded view is given in Fig. 7.

The magnet is operated by a bipolar power supply, capable of running a degaussing procedure, eliminating

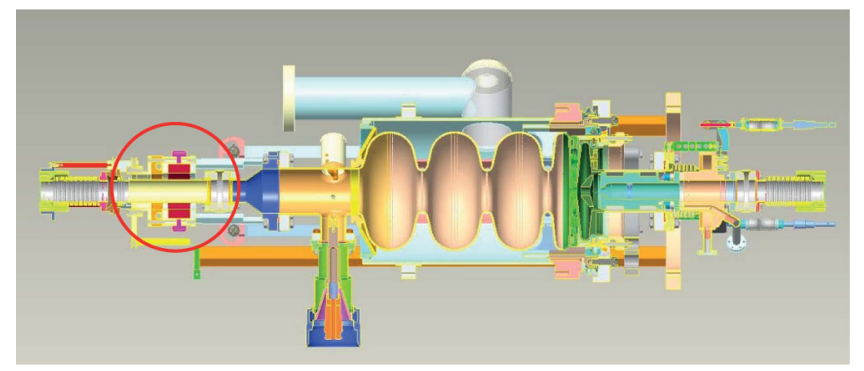

FIG. 6. The $3-1 / 2$ cell cavity is contained in titanium vessel filled with liquid helium. The SC solenoid is installed around the beam tube in front of the resonator (red circle) and connected to the same helium reservoir supplying the cavity vessel, the connecting lines transporting the liquid helium can be seen in Fig. 8.

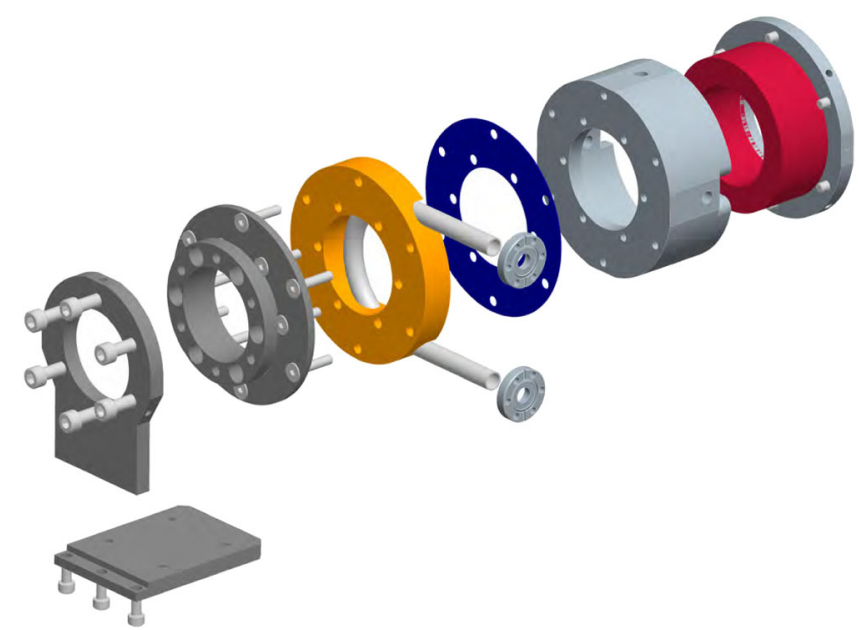

FIG. 7. Exploded drawing of the solenoid [17], from left to right: Support, main bracket, copper cooling block (orange), indium disc, iron yoke, SC coil (red), and yoke back plate. 




FIG. 8. Fully dressed SC solenoid with shielded steppers, main magnetic shield around the yoke, installed temperature sensors, isolated helium connection lines, and thermal anchors of the connecting (black and red) and measuring (cyan and blue) leads. The picture was taken after the insertion of the cold mass into the gun cryostat.

remanent fields in the iron yoke which may affect the niobium cavity during cool down. In addition, a passive magnetic shield out of CRYOPERM ${ }^{\circledR}$ was installed around the solenoid's yoke. The shield serves as another barrier to keep magnetic fields from reaching the superconducting cavity. As the solenoid is mounted on an x-y-stage it is possible to compensate for misalignment of the geometrical and magnetic axes. The steppers of this platform, containing permanent magnets, have also been shielded. All magnetic shields mounted together as well as the liquid helium supply lines, are shown in Fig. 8.

\section{SIMULATION STUDIES}

In order to visualize the possible gain in terms of emittance compensation, simulations using ASTRA's [18] cylindrical grid space charge algorithm for the particle tracking and SUPERFISH [14] for the generation of the field files were carried out [19]. Consequently, all simulated fields are rotationally symmetric. These studies cover the operation with higher bunch charges, i.e., few hundred $\mathrm{pC}$, which are aimed for by the use of bulk $\mathrm{Mg}$ cathode tips. For all simulations an accelerating gradient of $8 \mathrm{MV} / \mathrm{m}$ was set for the cavity, as this matches the currently used gradient for stable CW operation [20]. The advantage of such numerical studies is that the impact of the two implemented compensation tools on the beam's transverse emittance as well as its

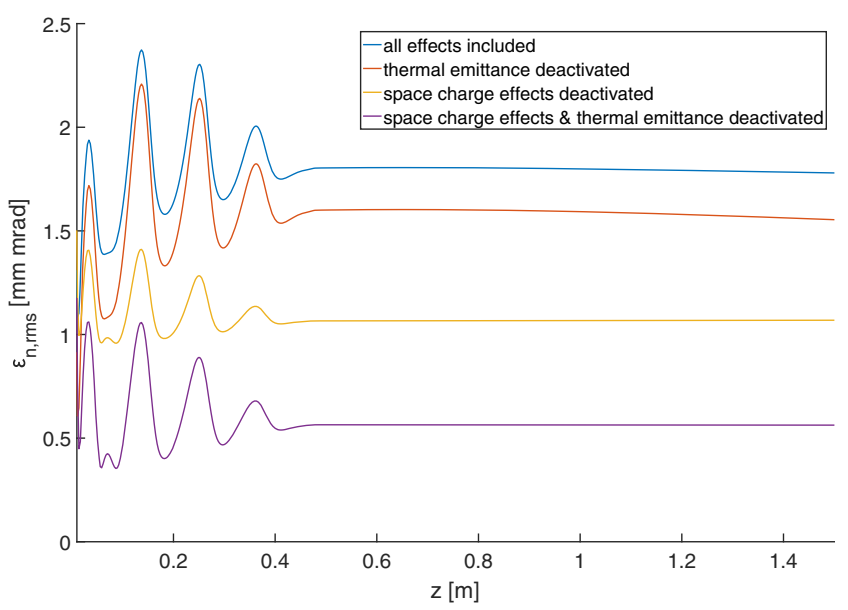

FIG. 9. Results of tracking a $100 \mathrm{pC}$ electron bunch through the ELBE SRF Gun with different emittance contributions generated with Astra. The bottom purple curve shows the baseline contribution of the rf field itself.

transverse expansion in particular can be analyzed separately and easily optimized for any chosen model environment. For the theoretical optimization analysis, a ideal homogeneous particle distribution was chosen at the beginning of the bunch generation. This corresponds to a flattop intensity distribution of the injector laser, which has not been realized yet as shown below, however, is intended to be implemented. Figure 9 shows the separation of different contributions to the emittance in the model environment for a $100 \mathrm{pC}$ bunch, where space charge begins to dominate. Examples of the code used are given in [21].

In order to allow for a comparison with experiment, the tracking results were (usually) analyzed up to $2.8 \mathrm{~m}$, matching the position of the SRF injector's analysis station



FIG. 10. Tracking of a $250 \mathrm{pC}$ bunch through the ELBE SRF Gun and along the first 2.8 meters of the beamline with deactivated solenoid. The dashed curves give the evolution of beam radius and transverse emittance for a case without rf focusing while the solid curves include optimization of the retraction of the photocathode in the half cell. 


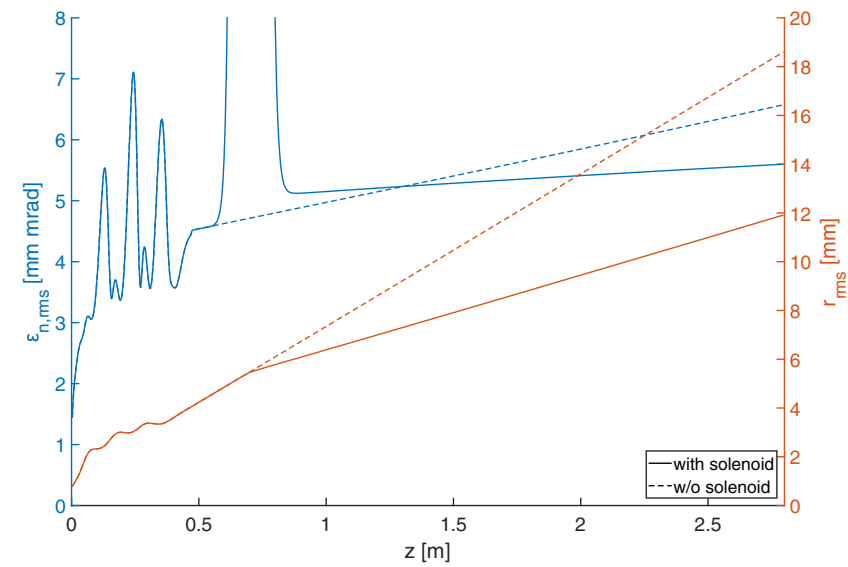

FIG. 11. Tracking of a $250 \mathrm{pC}$ bunch through the ELBE SRF Gun and along the first 2.8 meters of the beamline without rf focusing. The dashed curves give the evolution of beam radius and transverse emittance without active SC solenoid after the resonator while for the solid curves the solenoid field was optimized for a minimum emittance at the end. The strong peak in the emittance curve at the solenoid's location describes the temporary blowup of the transverse trace space caused by the rotation of the electron bunch in the magnet.

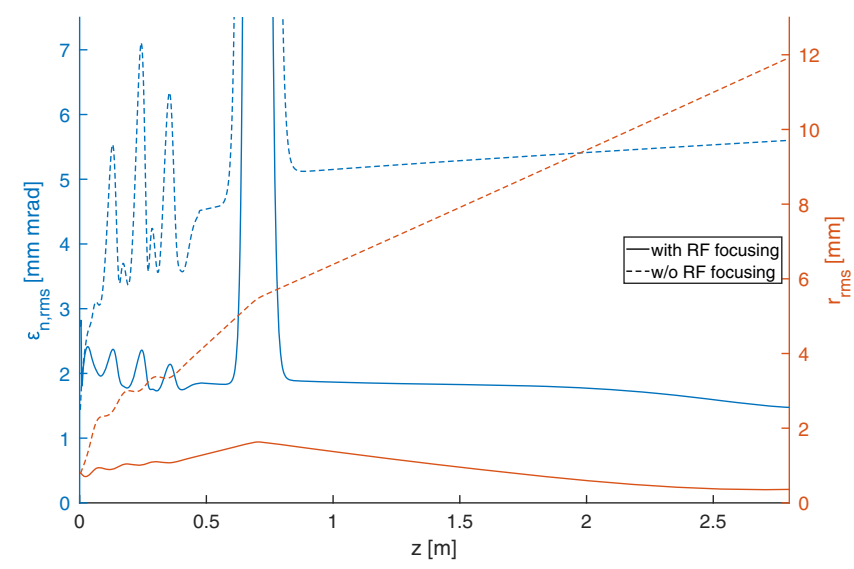

FIG. 12. Tracking of a $250 \mathrm{pC}$ bunch through the ELBE SRF Gun and along the first 2.8 meters of the beamline. The dashed curves give the evolution of beam radius and transverse emittance for an optimized solenoid field without rf focusing, corresponding to the solid curves in Fig. 11. The solid lines represent the result of the combination of $\mathrm{rf}$ focusing and the $\mathrm{SC}$ solenoid at the gun's exit.

in the diagnostics beamline. To give a representation of the findings of these comprehensive studies within the scope of this publication, selected tracking results for a bunch charge of $250 \mathrm{pC}$ were chosen to illustrate the benefits of the described compensation schemes.

An optimization of the initial input parameters, in particular the cathode's displacement, i.e., the strength of the $\mathrm{rf}$ focusing, with respect to the transverse emittance at $2.8 \mathrm{~m}$ from the cathode for this case yields a normalized RMS emittance of $\varepsilon_{\mathrm{n}, \mathrm{rms}}=1.86 \mathrm{~mm}$ mrad as compared to
TABLE I. Simulation Results for the tracking of a $250 \mathrm{pC}$ bunch from the SRF Gun.

\begin{tabular}{lcccc}
\hline \hline Mode & $\begin{array}{c}\varepsilon_{\mathrm{n}, \mathrm{rms}} \\
{[\mathrm{mm} \text { mrad }]}\end{array}$ & $\begin{array}{c}r_{\mathrm{rms}} \\
{[\mathrm{mm}]}\end{array}$ & $\begin{array}{c}\text { Cathode displacement } \\
{[\mathrm{mm}]}\end{array}$ & $\begin{array}{c}I_{s} \\
{[\mathrm{~A}]}\end{array}$ \\
\hline $\begin{array}{l}\text { No } \\
0\end{array}$ & optimized & 7.9 & 13.6 & 0 \\
RF & optimized & 1.9 & 5.4 & -3.9 \\
0 & & & & \\
Sol & optimized & 5.6 & 11.9 & 0 \\
2.3 & & & & \\
RF + Sol & 1.4 & 0.4 & -3.9 & 3.9 \\
\hline \hline
\end{tabular}

one of $7.92 \mathrm{~mm} \mathrm{mrad} \mathrm{for} \mathrm{the} \mathrm{same} \mathrm{parameters} \mathrm{without} \mathrm{rf}$ focusing. However, as the evolution of the bunch's attributes shown in Fig. 10 indicate, this optimization does not result in a proper focusing of the beam itself at this location.

A very similar result is achieved by simulation of the SC solenoid as the sole emittance compensation mechanism, as given in Fig. 11. Both the emittance and beam diameter are reduced by the solenoid, but, due to the placement of the magnetic lens behind the SC resonator, the observed reduction is much lower. The smallest emittance value which can be generated at the reference point is about $5.6 \mathrm{~mm} \mathrm{mrad}$ with a solenoid current of $2.3 \mathrm{~A}$. The compensation of the beam expansion is consequently insufficient to yield a clear focus.

However, if the scan of the solenoid field strength is repeated in simulation for an electron bunch already adequately manipulated by rf focusing, i.e., using the optimization results gained earlier, a much more favorable trace space distribution can be achieved at a solenoid current of 3.9 A, as depicted in Fig. 12. Previously, we have shown in more detailed analysis of the corresponding trace space distributions in [21] that the early focusing in the half cell also enables better compensation by the magnetic lens right after the gun resonator. Only by the combination of these two methods of compensation a proper reduction of the projected emittance and beam radius for bunch charges in the range of a few hundred $\mathrm{pC}$ can be accomplished at a reasonable distance from the injector. The numerical results for the chosen tracking studies are given in Table I.

\section{EXPERIMENTAL RESULTS}

The experimental tests of the described compensation schemes were conducted with bulk magnesium cathodes in the ELBE SRF Gun II. As this cathode material offers a moderate quantum efficiency-about $0.2 \%$ after laser cleaning of the oxide surface-the extraction of bunch charges larger $100 \mathrm{pC}$ was possible. However, at the time of these measurements the transverse intensity distribution of the used UV drive laser was not fully homogeneous. This is caused primarily by the difficulties to maintain a proper conversion efficiency of the IR light to fourth harmonic UV at a high repetition rate. To provide sufficient power to the 


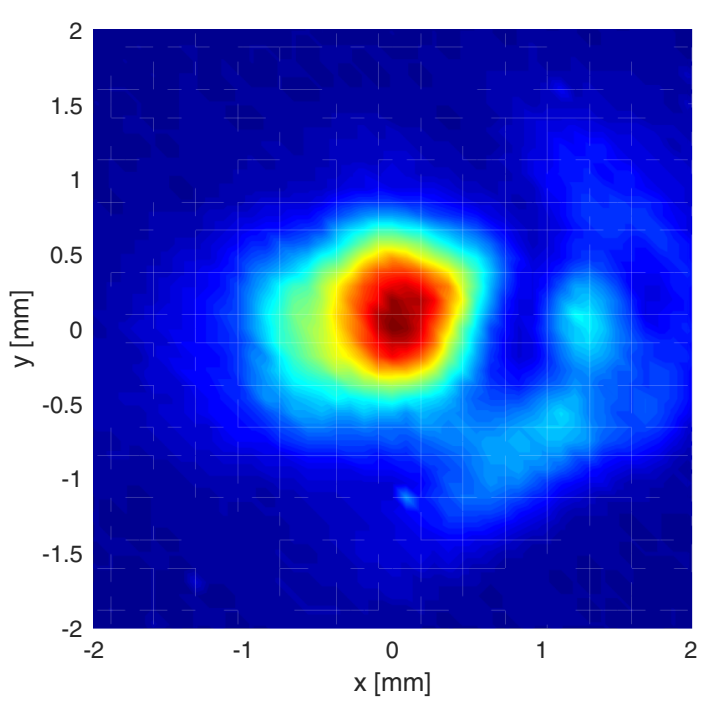

FIG. 13. An example intensity distribution of the UV laser recorded with a virtual cathode at the exact same position from the laser generator to the actual magnesium cathode: In order to maintain the required power for high bunch charge generation over the time of a measurement, the laser beamline especially the UV conversion section had to be realigned, thus changing the exact shape of the distribution as well. Compare Fig. 14 for a numerical representation.

photocathode, a further spatial filtering is not possible. Therefore, the generated electron bunch also starts with a distorted, non Gaussian charge distribution. An example of such a distribution is given in Fig. 13. This distribution then gets transformed by the electric and magnetic fields, respectively, of the 3.5 cell resonator as well as the following solenoid. Any asymmetries in these fields, consequently cause further distortion i.e., deviation from the ideal case. A proper alignment of cavity and magnet to avoid such asymmetries, can only be done by geometrical means at room temperature and may vary in the cooled-down state.

As the numerical simulation package AsTRA only generates symmetrical charge distributions as input for the tracking calculations, it is not possible to reproduce the actual charge distribution, which is finally the result of the interaction of the asymmetric laser intensity and the possibly inhomogeneous quantum efficiency of the cathodes surface. Hence, a deviation from the idealized situation of the numerical model is to be expected.

An additional disadvantage of the experimental situation versus the numerical model is that the individual study of the two compensation mechanisms is not fully possible for larger bunch charges as a minimum focusing is always required to fully capture the bunch by the used diagnostics. However, dedicated measurements of the contribution of the manipulation of certain parameters can still be done. Figure 15 summarizes the results of such a test for the cathode displacement in the case of a $120 \mathrm{pC}$ bunch.

Both, the measured beam diameter and the transverse emittance follow the predicted trend. While the earlier

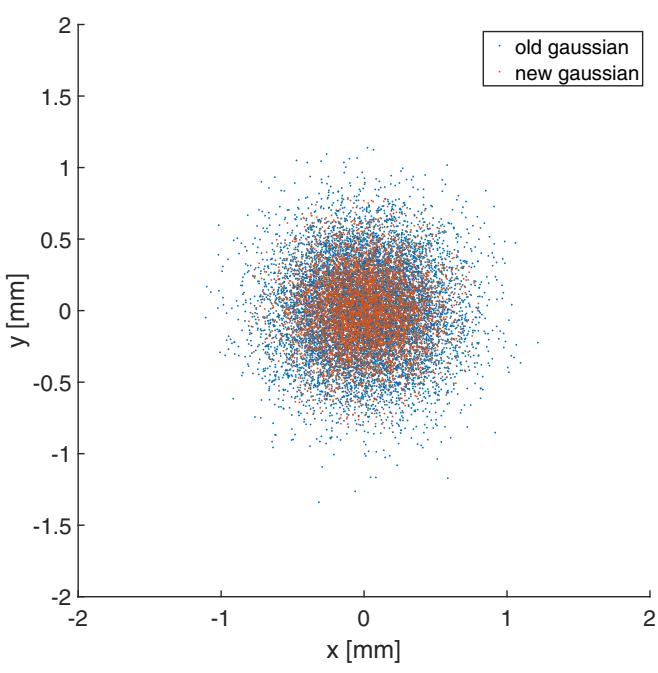

FIG. 14. Examples of numerically generated initial charge distributions for the simulation of the measurement done with the laser distribution depicted in Fig. 13, "old Gaussian" represents a regular $3 \sigma$ Gaussian distribution, while the "new Gaussian" was generated with a $15 \sigma$ environment. Thus the latter one also covers low density regions of the transverse laser intensity disproportionately, however, yields results closer to the one of the actual measurement as it sort of compensates for outlying parts of the laser distribution, compare Fig. 16. The graphical representation given here was generated with far less pseudoparticles than the actual simulation.

described cathode tuner was used to vary the displacement, the solenoid was kept at a fixed field strength allowing for the diagnosis of the beam on the corresponding profiler. Unfortunately, a further displacement of the photocathode caused a strong increase of the generated dark currentpotentially due to the activation of a field emitter close to the cathode. As a result, further measurements could not be made in this experimental study.

Similar to the above approach, the solenoid's compensation was examined by scanning the field strength via the solenoid current $I_{s}$ while keeping all other parameters fixed. The transverse emittance results for a different test with a bunch charge of $160 \mathrm{pC}$ are given in Fig. 16. The minimal normalized emittance is generated at about 3.5 A. By using an enlarged Gaussian as given in Fig. 14, which again does not fully agree with the actual charge distribution, a better agreement of simulation and measurement can at least be achieved for the stronger focused electron bunches. Despite the deviations from the numerically computed values in the ideal symmetric environment, the measured value of $\varepsilon_{\mathrm{n}, \mathrm{rms}}=5.7 \pm 0.5 \mathrm{~mm} \mathrm{mrad}$ demonstrates the successful handling of the beam dynamics by the combination of RF focusing and solenoid. An extended discussion of the measurement errors can be found in [21]. In comparison, the alternative thermionic injector of the ELBE accelerator provides bunch charges of $77 \mathrm{pC}$ at an emittance of about $\varepsilon_{\mathrm{n}, \mathrm{rms}} \approx 10 \mathrm{~mm}$ mrad. Hence, the SRF 


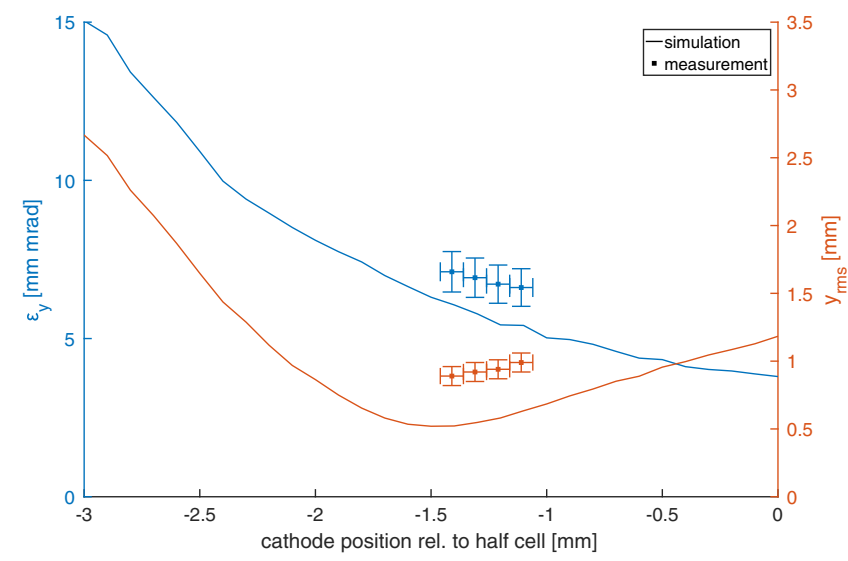

FIG. 15. Measurement of transverse emittance and beam size for different cathode positions for a bunch charge of $120 \mathrm{pC}$ : The measurement was conducted at an accelerating gradient of about $8 \mathrm{MV} / \mathrm{m}$ resulting in a kinetic energy of roughly 3.9 MeV. Since during the measurement a strong field emitter was activated, only four positions could be evaluated.

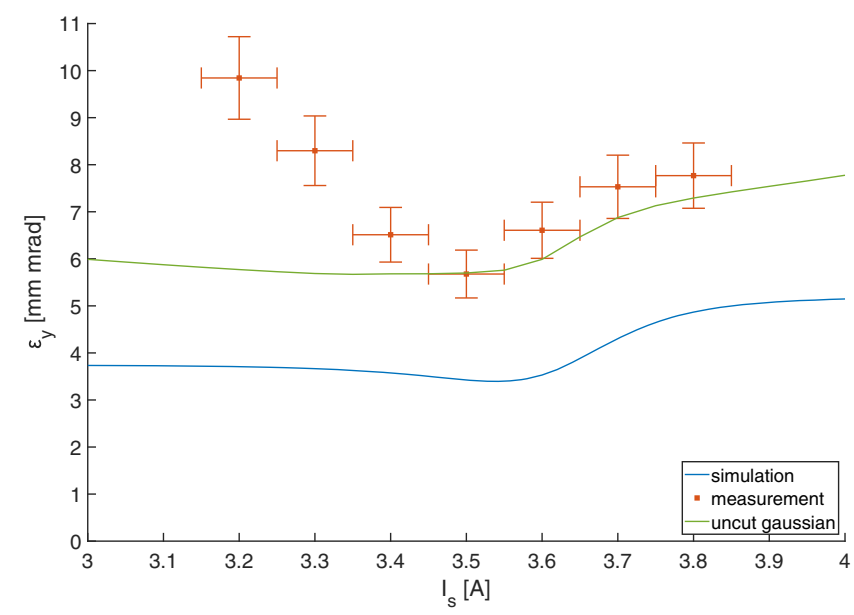

FIG. 16. A solenoid scan for a bunch charge of $160 \mathrm{pC}$ at an accelerating gradient of about $7.2 \mathrm{MV} / \mathrm{m}$ : Varying the solenoid current clearly affects the transverse emittance. However, especially the results for lower currents, i.e., less focusing deviates significantly from simulation. This is most likely caused by the larger contribution of outside particles and the hence enhanced beam radius to the total emittance. Using an uncut Gaussian for the initial charge distribution yields better agreeing simulation results, however, still lacks agreement for the lower current results.

Gun is able to deliver more than twice the bunch charge at about half the emittance.

\section{CONCLUSION}

SRF injectors present a promising concept for future accelerator based experiments, offering both, high quality beam parameters and increased duty cycle. Hence, they are a particularly interesting alternative to DC electron sources for
CW machines such as the ELBE accelerator at HZDR. Further examples for their use are the mentioned BERLinPro energy recovery linac, the LCLS-II accelerator at Stanford which recently received the formerly Wisconsin SRF gun for an injector upgrade, or the SRF injector at the BNL RHIC. The local SRF Gun project at Rossendorf has introduced two compensation schemes to its injector prototype to meet the challenge of emittance handling for increased bunch charges. These are rf focusing at the cathode in the resonator and the integration of an SC solenoid into the injector module. Both schemes were studied in simulation and experiment and proved in combination to provide a substantial reduction of the transverse emittance.

Currently, the biggest challenges of the project are the UV drive laser and the photocathodes. The laser requires improvements in its stability, output power, and homogeneity. The latter one referring to the transverse distribution, which is again linked to the power, as an increase in overall available intensity would enable further filtering to gain a symmetric intensity distribution [22]. In the case of the photocathodes, an enhancement of the quantum efficiency is possible through the use of semiconductor cathodes. However, the installation and operation of $\mathrm{Cs}_{2} \mathrm{Te}$ cathodes presented itself to be more complicated than expected and has not been satisfactorily realized so far.

Besides the ones discussed, further emittance compensation schemes for an SRF injector are being examined at HZDR. A description of the first successful test of the excitation of a transverse electric mode with a longitudinal magnetic component simultanious to the acceleratingtransverse magnetic - mode in a gun cavity at TJNAF can be found in [21].

When compared to state-of-the-art representatives of normal conducting injectors-for DC injectors the Cornell injector [23] is a good example, while for $\mathrm{rf}$ injectors the PITZ gun [24] may be referred to, SRF injectors are still inferior in terms of emittance compensation. However, as this publication shows, there are ways to overcome the increased challenges of this task in an SRF environment and further research and improvement are most likely to end up with equivalent levels of compensation with the advantage of SRF CW operation capabilities.

As the research within the ELBE SRF Gun project is in the process, a new project was launched at HZDR. The Dresden Advanced Light Infrastructure (DALI) is supposed to act as the successor of the ELBE facility, providing several $\mathrm{CW} \mathrm{THz}$ and further secondary particle sources. Within the framework of the preparatory studies of this project, a dedicated gun lab for further experiments with SRF injectors is envisaged, providing new dedicated resources for the continued injector development.

\section{ACKNOWLEDGMENTS}

We would like to thank for the help of all the people at the ELBE accelerator at HZDR, especially the people of the 
shift crews. Special thanks go to F. Roscher for his work at HZDR in '14/'15. In addition, we acknowledge the support of the European Community-Research Infrastructure Activity under the FP7 program (EuCARD-2, Contract No. 312453), as well as the support of the German Federal Ministry of Education and Research Grant No. 05K12CR1.

[1] A. Michalke, Photocathode inside superconducting cavities, Wuppertal, Germany, PhD thesis (1993-updated English translation 2009).

[2] T. Kamps, Status of GunLab: The R\&D infrastructure at HZB to facilitate CW SRF photoinjector development Berlin, PITZ Collaboration Meeting, Berlin, Germany (2014).

[3] T. Konomi et al., Design, fabrication and performance of SRF-Gun cavity, SRF, Vancouver, Canada, Proceedings (2015), http://accelconf.web.cern.ch/accelconf/srf2015/ papers/thpb059.pdf.

[4] J. Hao et al., Development of DC-SRF injector at Peking University, IPAC, Richmond, USA, Proceedings (2015), http://accelconf.web.cern.ch/AccelConf/IPAC2015/papers/ wepmn013.pdf.

[5] J. Sekutowicz, SRF gun development overview, $S R F$, Vancouver, Canada, Proceedings (2015), http://accelconf .web.cern.ch/accelconf/srf2015/papers/thaa02.pdf.

[6] S. Niles, Design and analysis of an eectron gun/booster and free electron laser optical theory, Ph.D. thesis, Naval Postgraduate School, 2010, http://www.dtic.mil/dtic/tr/ fulltext/u2/a531479.pdf.

[7] Q. Wu et al., Developing quarter wave SRF cavities for hadron colliders, SRF, Paris, France, Proceedings (2013), https://accelconf.web.cern.ch/AccelConf/SRF2013/papers/ frioa02.pdf.

[8] C. Boulware et al., Electron sources for future lightsources, ICFA Workshop on Future Light Sources, Newport News, VA (2012).

[9] F. Gabriel, P. Gippner, E. Grosse, D. Janssen, P. Michel, H. Prade, A. Schamlott, W. Seidel, A. Wolf, and R. Wünsch, The Rossendorf radiation source ELBE and its FEL projects, Nucl. Instrum. Methods Phys. Res., Sect. B 161-163, 1143 (2000).
[10] J. Teichert et al., First beam characterization of SRF Gun II at ELBE with a $\mathrm{Cu}$ photocathode, ERL, Stony Brook, USA, Proceedings (2015).

[11] A. Arnold et al., Commissioning results of the 2nd 3.5 cell SRF gun for ELBE, LINAC, Geneva, Switzerland, Proceedings (2014).

[12] R. Xiang et al., $\mathrm{Cs}_{2}$ Te normal conducting photocathodes in the superconducting rf gun, Phys. Rev. Accel. Beams 13, 043501 (2010).

[13] V. Volkov and D. Janssen, rf focusing - An instrument for beam quality improvement in superconducting rf guns, Nucl. Instrum. Methods Phys. Res,. Sect. A 452, 34 (2000).

[14] J. H. Billen, Poisson Superfish, version 7.17, Report No. LA-UR-96-1834, 2006.

[15] A. Arnold, Simulation und Messung der Hochfrequenzeigenschaften einer supraleitenden Photo-Elektronenquelle, Ph.D. thesis, Fakultät für Informatik und Elektrotechnik der Universität Rostock, 2012.

[16] F. Roscher (private communications).

[17] Niowave, Operation Manual for HZDR SC Solenoid with Iron Yoke 2011 (unpublished).

[18] K. Floettmann, Astra manual v3.1, http://www.desy.de/ $\sim$ mpyflo/Astra_manual/Astra-Manual_V3.1.pdf.

[19] H. Vennekate et al., Transverse Emittance Compensation for the Rossendorf SRF Gun II, IPAC, Dresden, Germany, Proceedings (2014), http://inspirehep.net/record/1313846/.

[20] A. Arnold et al., RF Performance Results of the 2nd ELBE SRF Gun, SRF'15, Vancouver, Canada (2015), http:// accelconf.web.cern.ch/accelconf/srf2015/papers/thpb055 .pdf.

[21] H. Vennekate, Emittance Compensation for SRF Photoinjectors (Wissenschaftlich-Technische Berichte HelmholtzZentrum Dresden-Rossendorf, 2017), HZDR-081.

[22] F. Roscher, Erprobung und Evaluierung eines räumlichen Lichtmodulators (SLM) zur Verbesserung der Strahlqualität eines Photokathodenlasers, Hochschule für Technik und Wirtschaft Dresden, Diploma thesis, 2016.

[23] A. Bartnik, C. Gulliford, I. Bazarov, L. Cultera, and B. Dunham, Operational experience with nanocoulomb bunch charges in the Cornell photoinjector, Phys. Rev. Accel. Beams 18, 083401 2015).

[24] G. Vashchenko et al., Recent Electron Beam Optimization at PITZ (FEL, Basel, 2014). 